\title{
Baseball orbital implants: a review of 39 patients
}

\author{
A G TYERS AND J R O COLLIN \\ From Moorfields Eye Hospital, City Road, London E C 1
}

SUMmARY Thirty-five secondary and six primary 'baseball' implants-acrylic balls in envelopes of donor sclera-were followed up for a minimum of 24 months. $97 \cdot 1 \%$ secondary and $50 \%$ primary implants were retained. Volume replacement was satisfactory in $83.8 \%$ with a single operation, and motility of the artificial eye was at least $14^{\circ}$ of horizontal gaze in $86 \%$, at least $7^{\circ}$ of upgaze in $95 \%$, and at least $14^{\circ}$ of downgaze in $62.6 \%$. Ptosis associated with the procedure occurred in $22 \%$, and $12 \cdot 2 \%$ developed a shallow fornix with instability of the artificial eye.

Removal of an eye results in a volume deficit in the orbit of about $7 \mathrm{ml}$, and the cosmetic effect of a perfectly made artificial eye may be spoiled by inadequate volume replacement. The volume of most prosthetic eyes is about $2 \mathrm{ml}$, and the simple addition of bulk to the prosthesis alone often fails to correct the enophthalmos and deep upper lid sulcus which develop invariably in adults and frequently in children. Such a bulky prosthesis cannot be supported indefinitely by the lower eyelid, which stretches, leading to the features of the "postenucleation socket syndrome'-enophthalmos, deep upper lid sulcus, lower lid laxity, and ptosis.

These uncosmetic changes can be reversed by sharing the required volume replacement between the prosthesis and a buried orbital implant placed within the muscle cone. Such an implant should be chemically and biologically inert, easy both to insert and to cover anteriorly with conjunctiva, and designed to incorporate the eye muscles to reduce migration within the orbit and to increase motility of the prosthesis.

Since Mules first implanted glass spheres 100 years ago an impressive variety of primary and secondary implants have been designed. Because the rectus muscles may be difficult to identify, once an anophthalmic socket has healed without an implant in situ, secondary implants often have no facility for attachment of the muscles. Recent examples include glass beads,' expandable silicone spheres, ${ }^{2}$ and RTV silicone. ${ }^{3}$ The baseball implant, an acrylic ball in an

Correspondence to A G Tyers, FRCS, Professorial Unit, Moorfields Eye Hospital, City Road, London EC1V 2PD. envelope of donor sclera, was described by Frueh and Felker. ${ }^{4}$ Although described as a secondary implant its design allows primary implantation as well, and it is equally effective whether or not the rectus muscles are found.

An $18 \mathrm{~mm}$ diameter sphere has a volume of $3.1 \mathrm{ml}$ but within a scleral covering the volume is about $4 \mathrm{ml}$, which with a light prosthesis is adequate to correct most cases of postenucleation socket syndrome.

\section{Patients and methods}

Forty-three implants were placed in 41 patients between March 1979 and March 1982. All had features of the postenucleation socket syndrome due to inadequate volume replacement in the orbit.

PREPARATION OF DONOR SCLERA

A donor eye with the cornea removed is everted and the ocular contents cleared away. The scleral envelope is stored in $70 \%$ alcohol in a refrigerator. When required the sclera is washed in six changes of saline over 24 hours and placed in an antibiotic such as framycetin sulphate 15 minutes before use.

\section{SURGICAL TECHNIQUE}

Adult patients are given a $100 \mathrm{mg}$ indomethacin suppository 12 hours before operation.

\section{Secondary implantation}

The fat in the muscle cone is exposed by making a horizontal conjunctival incision in the centre of the 
socket. This layer is dissected free from underlying Tenon's capsule as far as the fornices. Tenon's capsule is opened horizontally at the same level as the conjunctiva, and the intraconal fat should come into view. If an implant is found, it should be removed carefully to avoid damage to the rectus muscles and levator.

An attempt should be made to identify the rectus muscles. If found they are tagged with double armed 4 ' $\mathrm{O}$ ' chromic catgut sutures. An acrylic ball is selected which comfortably fills the space in the muscle cone. Too large an implant increases the risk of extrusion, reduces levator function, and makes the fitting of a satisfactory prosthesis difficult. Too small an implant provides inadequate volume replacement. We normally use a $16-20 \mathrm{~mm}$ ball. This is sewn into the envelope of donor sclera with long-acting absorbable sutures such as 6 ' $O$ ' polyglycolic acid sutures.

The scleral covered ball is placed in the muscle cone, and the medial, lateral, and inferior rectus muscles are attached in their normal orientation to the surface of the implant a few millimetres short of its anterior pole. If the superior rectus is also attached a ptosis may result from traction on the levator, so it is allowed to retract. After attachment of the muscles the double-armed sutures are brought through overlying Tenon's capsule and conjunctiva and tied. They should not be tied too far into the fornices or these will be shortened. If the muscles cannot be found, the three double-armed sutures are attached to the sclera at the same three sites and brought to the surface of the conjunctiva in the same way.

Tenon's capsule and conjunctiva are closed without tension in two layers with interrupted absorbable sutures.

\section{Primary implantation}

After enucleation the hole in posterior Tenon's capsule through which the optic nerve passed is opened widely to expose orbital fat. The muscles are marked with double-armed 4 ' $O$ ' chromic catgut sutures, and the operation proceeds as described above.

A clear glass or plastic shell is fitted to maintain the fornices, and we normally prescribe indomethacin $25 \mathrm{mg}$ tds for five days and topical chloramphenicol for two weeks postoperatively. The patient may be fitted with a temporary artificial eye in about six weeks and referred for impression fitting of the final prosthesis in three to six months.

Of the 43 implants in the series 24 were reexamined and 15 patients had their hospital records reviewed. Two patients had inadequate detail in their records and could not be traced for examination so were not included. A total of 35 secondary and six primary implants were reviewed in 39 patients. Attention was directed to retention of the implant, volume correction, motility of the prosthesis, and complications of the technique.

\section{Results}

RETENTION OF THE IMPLANT

Thirty-four of the 35 secondary implants $(97 \cdot 1 \%)$ were retained for 24-60 months' follow-up (average 40 months), and three of the six primary implants (50\%) were retained for 36-53 months' follow-up (average $44 \cdot 7$ months).

Table 1 Retained implants

\begin{tabular}{lll}
\hline $\begin{array}{l}\text { Months of } \\
\text { follow-up }\end{array}$ & Primary & Secondary \\
\hline $24-35$ & 2 & 13 \\
$36-47$ & 1 & 11 \\
$48-60$ & 3 & 10 \\
Total & - & 34 \\
\hline
\end{tabular}

The secondary implant which extruded was a $20 \mathrm{~mm}$ ball which became exposed at three days and was removed at 14 days. It had been used to replace a small Castroviejo implant removed at the same operation. The three primary implants were extruded at 8,14 , and 21 days respectively. All followed enucleation for recent trauma. Two were replaced with secondary baseball implants at six months and seven months respectively, and these have each been retained for 27 months to date. The retained primary implants followed enucleations for a painful blind eye (two cases) and choroidal malignant melanoma (one case).

In two secondary implants exposure occurred, but the implant was retained following resuture of the conjunctiva. One was a $20 \mathrm{~mm}$ ball, and resuture at 17 days was sufficient. The other was an $18 \mathrm{~mm}$ ball and required resuture at one month. Eighteen months later the implant became re-exposed, and a scleral patch was used to reinforce Tenon's capsule anteriorly. No further exposure has occurred after 17 months' follow-up.

\section{VOLUME CORRECTION}

If the extruded implants are omitted, 23 of the remaining 37 implants achieved full volume correction with a single operation. Fourteen patients $(37.8 \%)$ had residual enophthalmos or upper lid sulcus, but in only six $(16 \cdot 2 \%)$ was it necessary to supplement the volume with an orbital floor implant. Thirty-one $(83.8 \%)$ had a satisfactory result with a single operation. 
MOTILITY OF THE PROSTHESIS

A sample of 22 patients were re-examined specifically for motility of the artificial eye. Movement of the limbus was measured for horizontal gaze, upgaze, and downgaze.

Table 2 Motility of prosthesis

\begin{tabular}{llllc}
\hline $\begin{array}{l}\text { Limbal movement } \\
\text { mm }\end{array}$ & Horizontal & Upgaze & Downgaze & Total \\
\hline$<1$ & - & 1 & 1 & 2 \\
1 & 3 & 8 & 7 & 18 \\
2 & 7 & 7 & 4 & 18 \\
3 & 5 & 5 & 9 & 19 \\
$>3$ & 7 & 1 & 1 & 9 \\
\hline
\end{tabular}

With approximately $7^{\circ}$ being allowed for each millimetre, $86 \%$ achieved at least $14^{\circ}$ horizontal movement, $95 \%$ had at least $7^{\circ}$ upgaze, and $63.6 \%$ had at least $14^{\circ}$ downgaze.

\section{COMPLICATIONS}

Ptosis. Although it was not always recorded in the hospital notes we assume that almost all patients in the series had at least a minimal ptosis preoperatively as a feature of the postenucleation socket syndrome. In seven patients $(17 \cdot 1 \%)$ the preoperative ptosis was not corrected by orbital implantation and prosthetic adjustment alone. Six $(85.7 \%)$ of these required ptosis surgery.

Table 3 Analysis of patients with ptosis

\begin{tabular}{lcc}
\hline & Total & $\begin{array}{l}\text { Ptosis } \\
\text { surgery }\end{array}$ \\
\hline Ptosis corrected by implant alone & $? 25$ & - \\
Marked ptosis before and after implant & 7 & 6 \\
Marked ptosis after implant only & 0 & 6 \\
Total & 41 & $12(29.3 \%)$ \\
\hline
\end{tabular}

Nine patients $(22 \%)$ developed more marked ptosis after baseball implantation, and six $(66.7 \%)$ of these required surgical correction. All patients with ptosis had at least $5 \mathrm{~mm}$ of levator function. Three had a posterior levator resection, eight had an anterior levator resection, one had a FasanellaServat operation. There was no correlation between the severity of the ptosis and the age of the patient, the age at enucleation, the reason for enucleation, or the size of the implant.

Shallow fornices. Five patients $(12 \cdot 2 \%)$ with normal fornices before implantation developed a shallow fornix postoperatively. Of these only two $(40 \%)$ required subsequent fornix reconstruction with oral mucosa. Seven patients $(17 \%)$ had been noted to have shallow fornices before implantation, and these were usually worse postoperatively. Five of this group $(71.4 \%)$ required fornic reconstruction. Of the 12 patients with a shallow fornix after implantation the lower fornix alone was shallow in eight, the upper fornix alone in one, and both fornices in three.

Other complications. One implant, inserted into a grossly traumatised socket, migrated inferiorly and was removed electively at 34 months. A new baseball implant was placed at a higher level at the same operation and has been retained without incident for 11 months so far. Three patients developed conjunctival implantation cysts - two in the centre of the socket and one in the upper fornix. All were removed easily with direct closure of the conjunctiva.

\section{Discussion}

Apart from the minor inconvenience of preparing donor sclera the baseball implant is easy to prepare and insert. A moderate amount of postoperative oedema is common, as with all implants, and is reduced by indomethacin.

Selection of the correct size of acrylic ball is important. Too large an implant results in a shallow socket, poor fornices, and an increased risk of extrusion. Ocular prostheses should be at least $4 \mathrm{~mm}$ thick to achieve a realistic appearance, and this may not be possible if the convex posterior wall of the socket extends too far forward. A proptotic artificial eye may hinder lid closure, causing upper lid retraction, or the large implant may limit movement of the levator, causing ptosis. Too small an implant will not correct enophthalmos and a deep sulcus in the upper lid.

Extrusion is usually associated with oedema, haemorrhage, infection, or poor surgical technique and occurs most commonly within six months of surgery. The extruded primary implants in our series followed recent trauma and were probably due to oedema. We now avoid the baseball implant after enucleation for recent trauma. The single secondary implant which extruded was inserted at the time of removal of another implant. The $20 \mathrm{~mm}$ ball used may have been too large for the socket, or the trauma of removing the primary implant may have caused added oedema. Retention of the baseball implant is comparable with that of other buried implants, all of which have an extrusion rate of less than $4 \%$. Implanted sclera remains grossly unchanged for several years" and provides a stable pocket for the acrylic ball. There is some histological evidence that sclera gradually dissolves, ${ }^{9}$ but by then the pseudocapsule of adjacent tissues in the orbit provides adequate support.

If exposure of an implant occurs, resuture of the conjunctiva alone is often inadequate, though it was 
initially successful in our two cases. The use of a scleral patch ${ }^{10}$ extending almost to the fornices between Tenon's capsule and the conjunctiva is a better alternative and was successful when re-exposure occurred after 18 months in the second case.

Volume correction is excellent and lasting with the baseball implant and allows a light artificial eye to be fitted, which gains added support from the convex surface of the implant. When volume replacement was inadequate, it was noted as soon as postoperative oedema had settled. We have not observed significant late volume deficiency-for example, due to massive fat atrophy. Despite this some fat atrophy probably occurs, as with any socket surgery, and careful dissection should reduce this to a minimum. Significant undercorrection is best managed with a supplementary implant-usually a silicone block cut to size-introduced subperiosteally along the floor of the orbit via an incision in the lower lid.

Despite the apparent disadvantage of a 'ball and socket' joint between prosthesis and implant, motility was acceptable and comparable with that of the quasi-integrated implants. ${ }^{6}$ The good motility is probably due to movement of the fornices, well preserved because of the sutures between the muscles, implant, and conjunctiva.

Mild ptosis following enucleation is often corrected once the fulcrum over which the levator acts is raised by insertion of an implant. Inadequate size of implant fails to achieve this. Some ptoses do not improve and a few are made worse, probably owing to operative trauma to the levator and postoperative oedema. Postoperative ptosis may be improved by building up the prosthesis either on its anterior surface or upper rim. The failures are treated surgically. Levator resection is suitable for any ptosis with a levator function of more than $4 \mathrm{~mm}$. Less levator function that this should be treated with a brow suspension. Ptosis of $2 \mathrm{~mm}$ or less with a levator function of more than $12 \mathrm{~mm}$ can be treated with a Fasanella-Servat operation.

Shortened conjunctival fornices in a vertical direction may occur after enucleation unless care is taken with preservation and closure of conjunctiva. Insertion of an implant may exacerbate already shortened fornices, especially if it is too large. Closure of Tenon's capsule over a baseball implant may cause shortening of the fornices unless care is taken to dissect conjunctiva from Tenon's capsule as far as the fornices. Redundant conjunctiva in the centre of the socket is then avoided, and the fornices maintain their maximum possible depth. Despite this we usually avoid use of a baseball implant if the fornices are found to be short preoperatively. A dermofat graft $^{11}$ is a satisfactory alternative, though it tends to lose bulk during the first six months when used as a secondary implant. ${ }^{12}$ Conjunctiva grows over the anterior surface of a dermofat graft within six weeks. The same may occur if conjunctiva is recessed from the anterior pole of a scleral patch placed in front of a buried orbital implant, ${ }^{9}$ leaving more conjunctiva in the fornices. We have no experience of this and prefer to reconstruct a doubtful fornix with oral mucosa at the time of insertion of the implant if a baseball is to be used.

Implants that migrate usually do so in a downward and lateral direction, causing ectropion and loss of the lower fornix, with failure to retain the prosthesis. Minor degrees of migration may occasionally be managed with modification of the prosthesis, but often removal of the implant and insertion of a secondary implant is essential. The single case of migration in our series occurred in a grossly scarred socket.

The occurrence of conjunctival implantation cysts is well documented after enucleation surgery. ${ }^{13}$ They may occur within the orbit close to the implant or on the surface as in our series. They usually cause discomfort as they enlarge and may make wearing of the prosthesis impossible. Cysts close to the conjunctival surface are usually easy to remove. If there is doubt about the strength of the remaining layers anterior to the implant, the area should be reinforced with a patch of donor sclera between conjunctiva and Tenon's capsule.

\section{CONCLUSION}

The baseball implant can be recommended as a safe and convenient secondary implant and as the first approach to a volume deficit in the anophthalmic socket. It should probably be avoided if the conjunctival fornices are already shallow as a result of enucleation or other socket surgery.

This series of primary baseball implants is too small to allow us to draw valid conclusions. We now avoid this implant after recent trauma, but it may well prove to be of value in other cases.

\section{References}

1 Smith B, Obear M, Leone CR. The correction of enophthalmos associated with anophthalmos by glass bead implantation. Am J Ophthalmol 1967; 64: 1088-93.

2 Soll DB. Insertion of secondary orbital implant. Arch Ophthalmol 1973; 89: 214-6.

3 Vistnes LM, Paris GL. Uses of RTV silicone in orbital reconstruction. Am J Ophthalmol 1977; 83: 577-81.

4 Frueh BR, Felker GV. Baseball implant - a method of secondary insertion of an intraorbital implant. Arch Ophthalmol 1976; 94: 429-30.

5 Allen JH, Allen L. A buried muscle cone implant. Arch Ophthalmol 1950; 43: 879-90.

6 Spivy BE, Allen L, Burns CA. The Iowa enucleation implant. Am J Ophthalmol 1969; 67: 171-88.

7 Soll DB. Enucleation surgery - a new technique. Arch Ophthalmol 1972; 87: 197-7. 
8 Atkins AD, Roper-Hall MJ. Magnetic orbital implants. Br J Ophthalmol 1983; 67: 315-6.

9 Beyer CK, Albert DM. The use and fate of fascia lata and sclera in ophthalmic plastic and reconstructive surgery. The 1980 Wendell Hughes Lecture. Ophthalmology 1981; 88: 869-86.

10 Helveston EM. Human bank scleral patch for repair of exposed or extruded orbital implants. Arch Ophthalmol 1969; 82: 83-6.
11 Smith B, Petrelli R. Dermis fat graft as a moveable implant within the muscle conc. Am J Ophthalmol 1978; 85: 62-6.

12 Smith B, Bosniak S, Nesi F, Lisman R. Dermis-fat orbital implantation: 118 cases. Ophthalmic Surg 1983; 14: 941-3.

13 McCarthy RW, Bcyer CK, Dallow RL, Burke JF, Lessell S. Conjunctival cysts of the orbit following enucleation. Ophthalmology 1981; 88: 30-5. 